\title{
Long-term outcomes of uncemented or cemented arthroplasty revision following metal-on-metal total hip arthroplasty failure: A retrospective observational study with a mean follow-up of 7 years
}

Wenli Chen

Sun Yat-sen University First Affiliated Hospital

Mao Shuai

Sun Yat-sen University First Affiliated Hospital

Jinluan Lin

The Affiliated hospital of Fujian Medical University

\section{Baomin Chen}

Sun Yat-sen University First Affiliated Hospital

Mingdong Zhao ( $\square$ zhaonissann@163.com )

Jinshan Hospital, Fudan University

Xinchao Zhang

Jinshan Hospital, Fudan University

Weiguang Yu

Sun Yat-sen University First Affiliated Hospital

Guowei Han

Sun Yat-sen University First Affiliated Hospital

\section{Research article}

Keywords: Uncemented, Cemented, Total hip arthroplasty, Metal-on-Metal, Complication

Posted Date: March 12th, 2020

DOI: https://doi.org/10.21203/rs.2.20209/v2

License: (a) (i) This work is licensed under a Creative Commons Attribution 4.0 International License.

Read Full License 


\section{Abstract}

Background A high rate of metal-on-metal total hip arthroplasty (MoM-THA) has been well-known. The aim of this study was to compare the long-term outcomes of patients who had undergone uncemented or cemented THA(UTHA or CTHA) following initial MoM-THA failure. Methods Data from 234 patients (234 hips) who were treated with UTHA or CTHA following initial MoM-THA failure during 2007 - 2018 were retrospectively compared. Follow-up occurred 3 months, 6 months, 1 year, 2 years, and then every 1 year after conversion. The mean follow-up was 84.15 months (67-101 months). The primary endpoint was the Harris Hip Scores (HHS); secondary endpoint was the incidence of major orthopaedic complications. Results The HHS demonstrated statistically greater differences in Group CTHA than in Group UTHA 12 months after conversion. From the 12th month after conversion to the final follow-up, CTHA yielded superior functional outcomes than UTHA. Between-group noteworthy differences were observed regarding the rates of re-revision, aseptic loosening, and periprosthetic fracture (10.3\% for UTHA vs $2.5 \%$ for CTHA, $p=0.015 ; 16.3 \%$ for UTHA vs $5.9 \%$ for CTHA, $p=0.011$; and $12.0 \%$ for UTHA vs $4.2 \%$ for CTHA, $p=0.045$, respectively). Conclusion In the setting of revision following initial MoM-THA failure, we found definite evidence of the superiority of CTHA over UTHA in regard to improving functional outcomes and decreasing the incidence of major orthopaedic complications.

\section{Background}

Bearing surfaces provided by metal-on-metal total hip arthroplasty (MoM THA) became increasingly prevalent, particularly in the context of an ageing population, in earlier decades[1, 2]. In recent years, however, the use of MoM THA has sharply declined due to the reported low 10-year survivorship and high failure rates that are associated with a host of issues (i.e., adverse reactions to metal debris [ARMDs], aseptic loosening, and infection) [3, 4]. Failure after MoM THA is well recognised, is due to various reasons, and frequently requires revision intervention[5]. Among predominantly active individuals, failure that occurs secondary to MoM wear tends to be a concern[6]. Although MoM bearings have fallen out of favour as a result, orthopaedists continue to struggle with this issue of revision burden[7]. Poor bone stock may be attributed to the substantial bone and soft tissue destruction caused by ARMD that is powerfully implicated in the pathophysiology of MoM THA failure, contributing to the substantially high revision rate as well as the rapid time-to-failure[8].

This escalated MoM THA-related failure rate may also contribute to the increase in the use of uncemented or cemented total hip arthroplasty (UTHA or CTHA) [8, 9]. A limited number of studies have assessed complications due to the conversion of MoM THA to the use of uncemented or cemented femoral components (UTHA or CTHA)[3]. Interest in CTHA has increased over the last decade, with several studies showing higher Harris hip scores (HHS) with fewer orthopaedic complications for CTHA than for UTHA, while others have demonstrated no significant differences between the two[5].

Furthermore, there remain concerns that longer-term outcomes of UTHA may not be as robust as those of CTHA regarding reduced revision rates $[8,10]$. Additionally, the highly selected features of the studied 
patient data are common in the published literature[11, 12]. Consequently, the findings in those studies could not be referred to as valid.

To date, no definitive consensus exists on the long-term outcomes of conversion from primary MoM THA revision to UTHA or CTHA due to any cause[11]. Furthermore, given the lack of literature and in order to gain a better understanding of these types of conversion, we performed a retrospective study to assess the long-term outcomes of conversion from primary MoM THA to UTHA or CTHA.

\section{Methods}

\section{Study population}

A retrospective study was performed with an initial study cohort that included 326 patients (326 hips) identified from our joint registration database who underwent UTHA or CTHA revision due to prior primary MoM THA failure from March 2007 to January 2018. The main reasons for revision included ARMD, aseptic loosening, infection, dislocation, and fracture. The inclusion criteria for the study included patients who underwent a conversion procedure from primary MoM THA (Zimmer Biomet, Warsaw, IN) to UTHA or CTHA; all conversion procedures were performed by three experienced orthopaedists (WY, XZ, MZ) via a direct anterior approach, as reported[13]. Manufacturer details of stems and cups employed in UTHA or CTHA were shown in Table 1. The main exclusion criteria included patients without an MoMbearing surface at the time of conversion; patients with inadequate clinical data, active infection, dyskinesia, or bone-related diseases; patients unable to follow instructions; and patients with malignant tumours, injury severity scores (ISSs) $\geq 10$, brain dysfunction due to any cause within 6 months, pulmonary complications (i.e., reintubation), cardiovascular complications (i.e., cardiac arrest, myocardial infarction), renal complications (i.e., insufficiency or failure), vascular cognitive impairment, a history of alcohol and/or drug abuse, a body mass index $(\mathrm{BMI})>40 \mathrm{~kg} / \mathrm{m}^{2}$, and an American Society of Anesthesiologists (ASA) score of IV or V.

A standard protocol was utilised to obtain clinical and radiographic data. Follow-up occurred 3 months, 6 months, 1 year, 2 years, and then every 1 year after conversion. The primary endpoint was the modified HHS, which has scores ranging from 0 to 100 points, with higher scores representing better function. The secondary endpoints were the major orthopaedic complication rates. All the patients included underwent conversion to UTHA or CTHA at our medical centre and were assessed individually with the modified HHS at each follow-up. Image data were acquired at these same time points: anteroposterior (AP) radiographs of the pelvis and AP and lateral radiographs of the hip as well as computed tomography (CT) or magnetic resonance imaging (MRI) if necessary. The occurrence of major orthopaedic complications was recorded for each patient during the follow-up.

\section{Statistical analysis}

Between-group differences in terms of the baseline data and the main follow-up data were compared. Follow-up was calculated in days from the date of revision/conversion to the date of death or final follow- 
up, whichever occurred first. Revision was defined as the complete removal of the endoprosthesis. Rerevision was defined as the removal or exchange of any component. Prosthesis loosening and endoprosthesis failure were judged based on prior descriptions[8]. Heterotopic ossification was judged using the Brooker classification system[14]. Continuous data are presented as the means and standard deviations. T-tests were utilised to assess between-group differences if the data were consistent with assumptions regarding the normal distribution and homogeneity of variance. If not, Wilcoxon rank-sum tests were utilised. Categorical data are presented as frequencies and percentages and were compared between groups using Chi-squared tests or Fisher's exact tests, as appropriate. All statistical analyses were performed using SPSS, version 24.0 (IBM, Armonk, NY). A 2-sided $p$ value less than 0.05 was considered significant.

\section{Results}

Based on our criteria, a total of 234 individuals (234 hips) undergoing conversion from primary MoM THA to UTHA or CTHA were identified for the final analysis in the study (UTHA: $n=116$, mean age, $67.34 \pm 6.25$ years; CTHA: $n=118$, mean age, $67.45 \pm 6.21$ years). The mean time to failure after primary MoM THA was 4.3 years (1.2 - 6.5 years) for UTHA and 4.4 years ( 1.1 - 6.4 years) for CTHA. At the time of analysis, the mean follow-up time from conversion was 84.12 months (67- 100 months) for UTHA and 84.23 months (66-101 months) for CTHA. A study flow chart is presented in Figure 1, and the baseline data are shown in Table 2.

\section{Primary endpoint}

The mean HHS after conversion are shown in Table 3. The mean HHS in the UTHA and CTHA groups were $79.14( \pm 8.12)$ and $79.28( \pm 7.66) 3$ months after conversion, $86.65( \pm 6.62)$ and $87.76( \pm 7.44) 6$ months after conversion, $88.17( \pm 7.72)$ and $91.43( \pm 8.52) 12$ months after conversion, and 79.18 $( \pm 11.12)$ and $84.32( \pm 10.35)$ at the final follow-up, respectively. The HHS demonstrated statistically greater differences in the CTHA group than in the UTHA group 12 months after conversion $(p=0.031)$. From the 12th month after conversion to the final follow-up, CTHA yielded better functional outcomes than UTHA (all $p<0.05$ ). Almost $76 \%$ of the patients who experienced MoM THA failure and underwent conversion to UTHA or CTHA had acceptable HHS at the final follow-up. Differences in HHS were not significant between the groups 3 months or 6 months after conversion.

\section{Secondary endpoint}

There were 83 major orthopaedic complications in 116 UTHA patients versus 47 in 118 CTHA patients. Of the 83 UTHA-related orthopaedic complications, 12 (10.3\%) involved re-revision, 19 (16.3\%) were associated with aseptic loosening, 14 (12.0\%) involved periprosthetic fractures, and 6 (5.1\%) involved unbearable hip pain. Of the 47 CTHA-related orthopaedic complications, $3(2.5 \%)$ involved re-revision, 7 (5.9\%) were associated with aseptic loosening, 5 (4.2\%) involved periprosthetic fractures, and 8 (6.7\%) involved intolerable hip pain (Table 4). The between-group difference in the re-revision rate was significant at the last follow-up (10.3\% for UTHA vs $2.5 \%$ for CTHA, $p=0.015)$. Almost $82.1 \%$ of the cases 
of re-revision for UTHAs were attributed to aseptic loosening compared to $73.5 \%$ of the cases of rerevisions for CTHAs $(p=0.034)$. The mean time from revision to re-revision was 3 years $(1.4-3.5$ years $)$ for UTHA and 3 years (1.2 - 3.6 years) for CTHA $(p=0.154)$.

\section{Discussion}

The current findings provide evidence that the revision of primary MoM THA failure using CTHA results in superior long-term clinical outcomes compared with the use of UTHA. To our knowledge, this is the largest study regarding outcomes due to conversion after MoM THA failure.

Complications of MoM THA related to ARMD can result in significant bone and soft tissue destruction as well as increased metal ion levels, especially cobalt and chromium, potentially increasing the risk of implant failure and posing a challenge for future revision[8, 5]. Metal ions can inhibit osteoblast gene expression, and they have a negative impact on osteoblast cell number and activity $[3,7,15]$. Hence, this could ultimately result in bone ingrowth failure in the uncemented components utilised during the conversion to UTHA after MoM THA failure[15]. The results of MoM THA revision have revealed high rates of orthopaedic complications due to aseptic loosening, deep infection, and dislocation[3]. In the 2018 National Joint Registry Annual Report, the 14-year cumulative probability of revision was $22.2 \%$ for uncemented stemmed MoM THA[16]. Additionally, in the 2018 Australian Orthopaedic Association's Annual Report, the 15-year cumulative probability of revision was $29.6 \%[16]$. Whether increases in hip stability exist following MoM THA revision has become one of the key indicators $[8,17,18]$. Hip stability following conversion to CTHA is superior to that following conversion to UTHA owing to the instability of the bone and uncemented components[8]. There is often macroscopic damage or bone defects at the time of UTHA re-revision[15]. The cause of bone defects has been shown to be associated with malposition and a design that is too shallow for the acetabular component, resulting in atypically elevated wear triggered by edge loading[19, 1]. A prior study showed a high rate of aseptic loosening of the femur component after UTHA re-revision due to MoM THA failure[5]. Failure due to aseptic loosening occurs more frequently with UTHA re-revision than with CTHA re-revision[20]. The most appropriate rerevision intervention to decrease the high rate of aseptic loosening tends to be a matter of ongoing debate[8]. Perhaps there is an effective solution when both the femoral and acetabular components are well ingrown.

While there have been continued advancements in prosthetic materials, the risks associated with conversion from MoM THA to UTHA or CTHA remain a substantial concern[15, 8, 3]. However, the obtainable literature on the outcomes of this type of conversion is lacking and contradictory[21, 4, 22]. A growing but still extremely limited body of literature has described the role of UTHA or CTHA revision in the setting of prior MoM THA failure and has demonstrated significant differences in clinical outcomes, although all the studies are limited by small sample sizes and/or short-term follow-up periods $[3,13,8]$. Undeniably, invasive revision procedures are associated with a high rate of orthopaedic complications[8]. However, we failed to detect noteworthy distinctions regarding the rates of major orthopaedic complications 12 months after conversion. Concerns have existed regarding whether these two types of 
conversion have substantial differences in long-term outcomes, including orthopaedic complication rates[8, 18,3]. In 2002, Sierra et al.[23] identified 132 cases of conversion from MoM arthroplasties to UTHA and reported a high rate of major orthopaedic complications (45\%), including a $13 \%$ aseptic loosening rate and a $9 \%$ dislocation rate, which were all higher than the rates found in this current study. In 2009, Eswaramoorthy et al[24] reported on 76 patients who underwent conversion from MoM arthroplasties to UTHA. Similar to the findings observed in the current study of failed MoM THA treatment, both aseptic loosening and periprosthetic fracture were the primary orthopaedic complications due to conversion. They also described a high rate of major orthopaedic complications (24\%), mainly attributable to a high rate of aseptic loosening (20\%). Then, Taheriazam et al.[25] assessed outcomes in 138 patients who were treated via conversion from MoM THA to CTHA and showed a major orthopaedic complication rate of $16 \%$ at the 2 -year follow-up, including a $16 \%$ aseptic loosening rate. Based on a similar premise, Stryker et al.[26] reported on 114 cases of conversion from MoM THA to CTHA and showed a major orthopaedic complication rate of $18 \%$, with a re-revision rate of $7 \%$, primarily attributable to aseptic loosening (14\%) and deep infection (6\%).

Femoral aseptic loosening, especially in young, active patients, was a common factor for re-revision after conversion in the current study, which was also found by others assessing UTHA or CTHA revisions. CTHA has been developed in an effort to improve the fusion of cement and bone tissue and has become conventional for MoM THA revision failure[3, 13]. Short-term or midterm results of CTHA conversion following MoM THA failure have revealed extremely low rates of major orthopaedic complications, especially aseptic loosening[20, 5, 27]. Rahman et al.[5] reported on 20 patients who experienced MoM THA failure and who underwent conversion using CTHA; few patients were found to have aseptic loosening.

This current analysis also reveals that the reason for conversion has a prevailing impact on the outcomes of conversion. With modern THA and surgical techniques, conversion due to an indication of MoM wear has low rates of re-revision, regardless of the use of UTHA or CTHA for conversion, whereas conversion due to conventional periprosthetic fracture tends to be associated with a higher rate of re-revision. It is imperative that these facts are understood by orthopaedists and patients prior to conversion.

It should be acknowledged that there are limitations in this study. First, selection bias appears to be unavoidable due to the exclusion of a number of patients. Second, this retrospective observational study was susceptible to errors in recording differences in comorbidities and orthopaedic complications, which may have created unaccounted confounding variables and may have resulted in a diminished power to draw convincing conclusions. Attempts were made to allow for more than a few confounding variables; nevertheless, we believe that this analysis is inadequate. Third, we failed to include data on metal ion concentrations as well as information about high- and low-volume orthopaedists. Despite these limitations, we believe that the margin of error is tolerable in the current setting because of the relatively large sample size.

\section{Conclusions}


The long-term results reported in this study support a growing body of evidence that conversion to CTHA due to primary MoM THA failure is associated with more significant improvements in modified HHS as well as lower major orthopaedic complication rates than conversion to UTHA.

\section{Abbreviations}

UTHA: uncemented total hip arthroplasty; CTHA: cemented total hip arthroplasty; MoM THA: metal-onmetal total hip arthroplasty; ARMD: adverse reaction to metal debris; BMD: bone mineral density; ISS: injury severity score; BMI: body mass index; ASA: American Society of Anesthesiologists; HHS: Harris hip scores; AP: anteroposterior; CT: computed tomography; MRI: magnetic resonance imaging; SD: Standard deviation.

\section{Declarations}

\section{Acknowledgements}

The authors would like to thank Hang Yu and Junxing Ye for help with retrieval of patients' data.

\section{Funding}

Funding for this research was received from the National Natural Science Foundation of China (Grant No. 81971315).

\section{Availability of data and materials}

The datasets used and/or analysed during the current study are available from the corresponding author upon reasonable request.

\section{Authors' contributions}

WC performed the data collection and analysis and participated in manuscript writing. SM, JL, and BC performed the database setup and statistical analysis. $M Z, X Z, W Y$, and $G H$ performed the operations. $M Z$ and $X Z$ participated in the study design and coordination and helped to draft the manuscript. All authors have read and approved the final manuscript.

\section{Ethics approval and consent to participate}

This study was approved by the Medical Ethics Committee (The First Affiliated Hospital, Sun Yat-sen University), and an exemption from informed consent was obtained from our responsible Investigational Ethics Review Board.

\section{Consent for publication}

Not applicable. 


\section{Competing interests}

The authors declare that they have no competing interests.

\section{References}

1. Vovos TJ, Lazarides AL, Ryan SP et al. Prior Hip Arthroscopy Increases Risk for Perioperative Total Hip Arthroplasty Complications: A Matched-Controlled Study. J Arthroplasty. 2019;34(8):1707-10.

2. Langton DJ, Jameson SS, Joyce TJ et al. Early failure of metal-on-metal bearings in hip resurfacing and large-diameter total hip replacement A CONSEQUENCE OF EXCESS WEAR. J Bone Joint Surg Br. 2010;92B(1):38-46.

3. Borton ZM, Mumith AS, Nicholls AJ et al. The Outcome of Revision Surgery for Failed Metal-on-Metal Total Hip Arthroplasty. J Arthroplasty. 2019;34(8):1749-54.

4. Tauriainen TJT, Niinimaki TT, Niinimaki JL et al. Poor Acetabular Component Orientation Increases Revision Risk in Metal-on-Metal Hip Arthroplasty. J Arthroplasty. 2017;32(7):2204-7.

5. Rahman WA, Amenabar T, Hetaimish BM et al. Outcome of Revision Total Hip Arthroplasty in Management of Failed Metal-on-Metal Hip Arthroplasty. J Arthroplasty. 2016;31(11):2559-63.

6. De Haan R, Campbell PA, Su EP et al. Revision of metal-on-metal resurfacing arthroplasty of the hip The influence of malpositioning of the components. J Bone Joint Surg Br. 2008;90B(9):1158-63.

7. Carlson BC, Bryan AJ, Carrillo-Villamizar NT et al. The Utility of Metal lon Trends in Predicting Revision in Metal-on-Metal Total Hip Arthroplasty. J Arthroplasty. 2017;32(9):S214-S9.

8. Crawford DA, Adams JB, Morris MJ et al. Revision of Failed Metal-on-Metal Total Hip Arthroplasty: Midterm Outcomes of 203 Consecutive Cases. J Arthroplasty. 2019;34(8):1755-60.

9. Park KS, Yoon TR, Song EK et al. Cementless Acetabular Socket Revisions Using Metasul Metal-onMetal Bearings. J Arthroplasty. 2010;25(4):533-7.

10. Robinson PG, Wilkinson AJ, Meek RMD. Metal ion levels and revision rates in metal-on-metal hip resurfacing arthroplasty: a comparative study. Hip Int. 2014;24(2):123-8.

11. Matharu GS, Pandit HG, Murray DW et al. Adverse reactions to metal debris occur with all types of hip replacement not just metal-on-metal hips: a retrospective observational study of 3340 revisions for adverse reactions to metal debris from the National Joint Registry for England, Wales, Northern Ireland and the Isle of Man. BMC Musculoskelet Disord. 2016;17:222.

12. Jameson SS, Baker PN, Mason J et al. Independent predictors of revision following metal-on-metal hip resurfacing A RETROSPECTIVE COHORT STUDY USING NATIONAL JOINT REGISTRY DATA. J Bone Joint Surg Br. 2012;94B(6):746-54.

13. Bouveau V, Haen TX, Poupon J et al. Outcomes after revision of metal on metal hip resurfacing to total arthroplasty using the direct anterior approach. Int Orthop. 2018;42(11):2543-8.

14. Rama K, Vendittoli PA, Ganapathi M et al. Heterotopic Ossification After Surface Replacement Arthroplasty and Total Hip Arthroplasty. J Arthroplasty. 2009;24(2):256-62. 
15. Zijlstra WP, Bulstra SK, van Raay J et al. Cobalt and chromium ions reduce human osteoblast-like cell activity in vitro, reduce the OPG to RANKL ratio, and induce oxidative stress. J Orthop Res. 2012;30(5):740-7.

16. Lainiala OS, Reito AP, Nieminen JJ et al. Declining Revision Burden of Metal-on-Metal Hip Arthroplasties. J Arthroplasty. 2019;34(9):2058-+.

17. Grechenig S, Gueorguiev B, Berner A et al. A novel locking screw hip stem to achieve immediate stability in total hip arthroplasty: A biomechanical study. Injury. 2015;46:S83-S7.

18. Pallaver $A, Z$ wicky $L$, Bolliger $L$ et al. Long-term results of revision total hip arthroplasty with a cemented femoral component. Arch Orthop Trauma Surg. 2018;138(11):1609-16.

19. Gross TP, Liu F. Outcomes After Revision of Metal-on-Metal Hip Resurfacing Arthroplasty. J Arthroplasty. 2014;29(9):219-23.

20. Fleischman AN, Tarabichi M, Magner Z et al. Mechanical Complications Following Total Hip Arthroplasty Based on Surgical Approach: A Large, Single-Institution Cohort Study. J Arthroplasty. 2019;34(6):1255-60.

21. Mueller LA, Schmidt R, Ehrmann C et al. Modes of Periacetabular Load Transfer to Cortical and Cancellous Bone after Cemented versus Uncemented Total Hip Arthroplasty: A Prospective Study Using Computed Tomography-Assisted Osteodensitometry. J Orthop Res. 2009;27(2):176-82.

22. Liow MHL, Dimitriou D, Tsai TY et al. Preoperative Risk Factors Associated With Poor Outcomes of Revision Surgery for "Pseudotumors" in Patients With Metal-on-Metal Hip Arthroplasty. J Arthroplasty. 2016;31(12):2835-42.

23. Sierra RJ, Cabanela ME. Conversion of failed hip hemiarthroplasties after femoral neck fractures. Clin Orthop Relat Res. 2002(399):129-39.

24. Eswaramoorthy VK, Biant LC, Field RE. Clinical and radiological outcome of stemmed hip replacement after revision from metal-on-metal resurfacing. J Bone Joint Surg Br. 2009;91B(11):1454-8.

25. Taheriazam A, Saeidinia A. Conversion to total hip arthroplasty in posttraumatic arthritis: short-term clinical outcomes. Orthop Res Rev. 2019;11:41-6.

26. Stryker LS, Odum SM, Fehring TK et al. Revisions of Monoblock Metal-on-metal THAs Have High Early Complication Rates. Clin Orthop Relat Res. 2015;473(2):469-74.

27. Tyson Y, Rolfson O, Karrholm J et al. Uncemented or cemented revision stems? Analysis of 2,296 first-time hip revision arthroplasties performed due to aseptic loosening, reported to the Swedish Hip Arthroplasty Register. Acta Orthop. 2019;90(5):421-6.

\section{Tables}

\section{Table 1 Manufacturer details of stems and cups employed in UTHA or CTHA}




\begin{tabular}{|c|c|c|}
\hline Patients, No. & Stem & Cup \\
\hline \multicolumn{3}{|l|}{ UTHA } \\
\hline 65 & Corail $^{1}$, & Reflection uncemented ${ }^{2}$, \\
\hline 51 & Filler ${ }^{3}$ & Trident $^{4}$, Igloo $^{3}$ \\
\hline \multicolumn{3}{|l|}{ CTHA } \\
\hline 69 & Exeter ${ }^{4}$ & Exeter $^{4}$, Elite $^{4}, \mathrm{IP} / \mathrm{SP} 1^{5}$, \\
\hline 49 & Spectron $E F^{2}$, & Contemporary $^{4}$, Marathon $^{1}$ \\
\hline
\end{tabular}

${ }^{1}$ DePuy, ${ }^{2}$ Smith \& Nephew, ${ }^{3}$ Biotechni, ${ }^{4}$ Stryker, ${ }^{5}$ Waldemar LINK. UTHA: uncemented total hip arthroplasty; CTHA: cemented total hip arthroplasty.

\section{Table 2 Patient demographics and outcomes}

\begin{tabular}{|c|c|c|c|}
\hline Variable & $\mathrm{UTHA}^{\mathrm{a}}(\mathrm{n}=116)$ & $\mathrm{CTHA}^{\mathrm{b}}(\mathrm{n}=118)$ & $\begin{array}{l}p- \\
\text { value }\end{array}$ \\
\hline Sex, $M / F$ & $52 / 64$ & $53 / 65$ & $0.409^{c}$ \\
\hline Age, years & $67.34 \pm 6.25$ & $67.45 \pm 6.21$ & $0.142^{\mathrm{d}}$ \\
\hline $\mathrm{BMI}, \mathrm{kg} / \mathrm{m}^{2}$ & $28.23 \pm 7.32$ & $28.15 \pm 7.22$ & $0.261^{\mathrm{d}}$ \\
\hline $\mathrm{BMD}$ & $-3.47 \pm 0.26$ & $-3.48 \pm 0.42$ & $0.132^{\mathrm{d}}$ \\
\hline Side, left/right & $56 / 60$ & $57 / 61$ & $0.277^{\mathrm{c}}$ \\
\hline $\begin{array}{l}\text { Interval to failure after primary MoM } \\
\text { THA (years) }\end{array}$ & $4.3(1.2-6.5)$ & $4.4(1.1-6.4)$ & $0.331^{\mathrm{d}}$ \\
\hline Comorbidities, n\% & & & $0.402^{\mathrm{e}}$ \\
\hline Hypertension & $61(52.5)$ & $69(58.4)$ & \\
\hline Diabetes mellitus & $32(27.5)$ & $28(23.7)$ & \\
\hline Hypertension and Diabetes mellitus & $23(20.0)$ & $21(17.9)$ & \\
\hline Mechanism of injury, $\mathrm{n} \%$ & & & $0.780^{\mathrm{e}}$ \\
\hline Traffic-related injury & $26(22.4)$ & $28(23.7)$ & \\
\hline Injury by falling & $51(43.9)$ & $46(38.9)$ & \\
\hline Tamp injury & $39(33.7)$ & $44(37.4)$ & \\
\hline ASA scale, $\mathrm{n} \%$ & & & $0.920^{\mathrm{e}}$ \\
\hline प & $22(18.9)$ & $24(20.3)$ & \\
\hline 0 & $64(55.1)$ & $63(53.3)$ & \\
\hline प & $30(26.0)$ & $31(26.4)$ & \\
\hline Preoperative HHS & $56.33 \pm 17.36$ & $56.43 \pm 16.92$ & $0.164^{\mathrm{d}}$ \\
\hline Follow-up period (months) & $84.12 \pm 16.62$ & $84.23 \pm 17.49$ & $0.214^{\mathrm{d}}$ \\
\hline
\end{tabular}

UTHA ${ }^{a}$ : uncemented total hip arthroplasty; $C T H A^{b}$ : cemented total hip arthroplasty; ${ }^{C}$ Analysed using the chi-square test; $d_{\text {Analysed using an independent-samples t-test; }}$ ${ }^{e}$ Analysed using the Mann-Whitney test. BMI: body mass index; BMD: bone mineral density;MoM THA: metal-on-metal total hip arthroplasty; ASA: American Society of Anesthesiologists; HHS: Harris hip scores.

Table 3 Long-term follow-up: functional outcomes 


\begin{tabular}{llll}
\hline HHS, month after conversion & $\mathrm{UTHA}^{\mathrm{a}}(\mathrm{n}=116)$ & $\mathrm{CTHA}^{\mathrm{b}}(\mathrm{n}=118)$ & $p$-value \\
\hline 3 & $79.14 \pm 8.12$ & $79.28 \pm 7.66$ & 0.212 \\
6 & $86.65 \pm 6.62$ & $87.76 \pm 7.44$ & 0.181 \\
\hline 12 & $88.17 \pm 7.72$ & $91.43 \pm 8.52$ & $0.031^{*}$ \\
\hline 24 & $88.72 \pm 7.35$ & $90.47 \pm 7.75$ & $0.036^{*}$ \\
\hline 36 & $87.14 \pm 8.43$ & $89.43 \pm 8.27$ & $0.027^{*}$ \\
\hline 8 & $87.56 \pm 9.42$ & $88.77 \pm 9.72$ & $0.025^{*}$ \\
\hline 72 & $86.32 \pm 9.68$ & $87.73 \pm 11.25$ & $0.014^{*}$ \\
84 & $82.29 \pm 10.16$ & $85.71 \pm 10.12$ & $0.011^{*}$ \\
Final follow-up & $79.78 \pm 11.65$ & $84.72 \pm 11.82$ & $<0.001^{*}$ \\
\hline
\end{tabular}

*Statistically significant values.

$U T H A^{a}$ : uncemented total hip arthroplasty; $C T H A^{b}$ : cemented total hip arthroplasty; HHS: Harris hip scores.

Table 4 Long-term follow-up: prosthesis-related complications

\begin{tabular}{llll}
\hline Variable, $\mathrm{n} \%$ & $\mathrm{UTHA}^{\mathrm{a}}(\mathrm{n}=116)$ & $\mathrm{CTHA}^{\mathrm{b}}(\mathrm{n}=118)$ & $p$-value \\
\hline Re-revision & $12(10.3)$ & $3(2.5)$ & $0.015^{*}$ \\
\hline Aseptic loosening & $19(16.3)$ & $7(5.9)$ & $0.011^{*}$ \\
\hline Periprosthetic fracture & $14(12.0)$ & $5(4.2)$ & $0.045^{*}$ \\
\hline Dislocation & $10(8.6)$ & $4(3.3)$ & 0.092 \\
\hline Femur shaft fracture & $3(2.5)$ & $3(2.5)$ & 0.983 \\
\hline Deep infection & $3(2.5)$ & $8(6.7)$ & 0.130 \\
Unbearable hip pain & $6(5.1)$ & $8(6.7)$ & 0.604 \\
\hline Lower limb shortening $(>1.5 \mathrm{~cm})$ & $5(4.3)$ & $2(1.6)$ & 0.240 \\
\hline Heterotopic ossification & $11(9.4)$ & $7(5.9)$ & 0.308
\end{tabular}

*Statistically significant values.

$U T H A^{a}$ : uncemented total hip arthroplasty; $C T H A^{b}$ : cemented total hip arthroplasty.

\section{Figures}


From March 2007 to January 2018, 326 patients (326 hips) were identified from our joint registration database who underwent an UTHA or CTHA revision due to a prior primary MoM THA

\begin{tabular}{|l|}
\hline -ISS $\geq 10(n=5)$ \\
-brain dysfunction $(n=4)$ \\
-pulmonary complications $(n=7)$ \\
-cardiovascular complications $(n=3)$ \\
-renal complications $(n=3)$ \\
-vascular cognitive impairment $(n=2)$ \\
-alcohol and/or drug abuse $(n=4)$ \\
-BMI $>40 \mathrm{~kg} / \mathrm{m} 2(n=11)$ \\
-ASA score of IV or V(n=4)
\end{tabular}

Reasons for exclusion $(n=92)$ -patients without a MoM bearing surface $(n=12)$ -inadequate clinical data $(n=9)$ -active infection $(n=7)$ -dyskinesia $(n=2)$ -bone-related diseases $(n=12)$ -inability to follow instruction $(n=3)$ -malignant tumour $(n=4)$

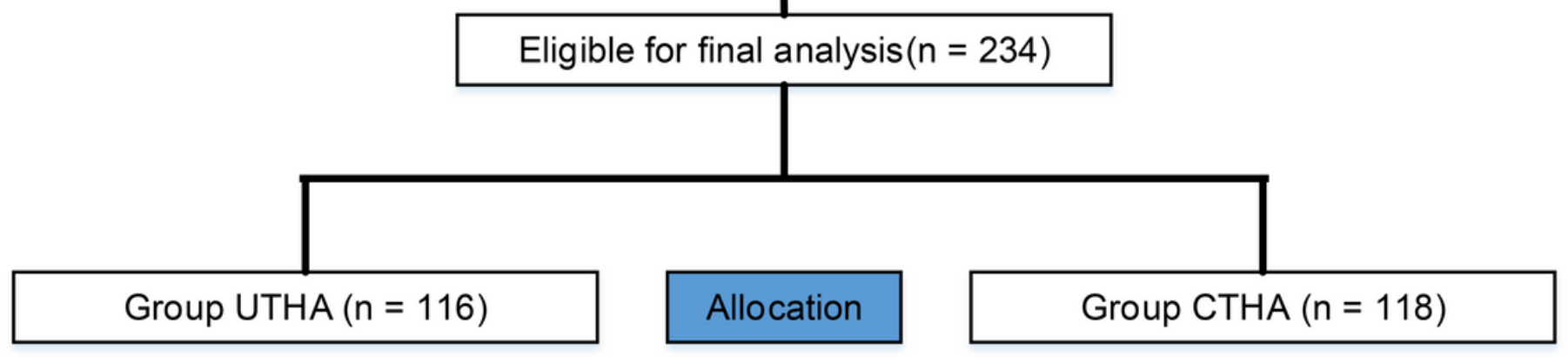

Figure 1

Flow diagram demonstrating methods for identification and exclusion of studies to compare the longterm outcomes of patients who had undergone an uncemented or cemented total hip arthroplasty (THA) revision for prior failed primary metal-on-metal THA (MoM THA). 